\title{
Multiple-view time-resolved diffuse optical tomography based on structured illumination and compressive detection
}

Andrea Farina, Marta Betcke, Nicolas Ducros, Laura di Sieno, Andrea Bassi, et al.

Andrea Farina, Marta Betcke, Nicolas Ducros, Laura di Sieno, Andrea Bassi, Antonio Pifferi, Gianluca Valentini, Simon Arridge, Cosimo D'Andrea, "Multiple-view time-resolved diffuse optical tomography based on structured illumination and compressive detection," Proc. SPIE 10412, Diffuse Optical Spectroscopy and Imaging VI, 1041203 (28 July 2017); doi: 10.1117/12.2290088 


\title{
Multiple-view time-resolved diffuse optical tomography based on structured illumination and compressive detection Andrea Farina ${ }^{1}$, Marta Betcke ${ }^{2}$, Nicolas Ducros ${ }^{3}$, Laura di Sieno ${ }^{4}$, Andrea Bassi ${ }^{1,4}$, Antonio Pifferi ${ }^{4}$, Gianluca Valentini ${ }^{1,4}$, Simon Arridge ${ }^{2}$, Cosimo D'Andrea

\author{
${ }^{1}$ Consiglio Nazionale delle Ricerche, IFN, Piazza L. da Vinci 32, 20133 Milano, Italy \\ ${ }^{2}$ Centre for Medical Image Computing, University College London, Malet Place, London \\ WC1E 6BT, United Kingdom \\ ${ }^{3}$ CREATIS, CNRS UMR5220, INSERM U1044, Université de Lyon, INSA Lyon, Villeurbanne, France \\ ${ }^{4}$ Politecnico di Milano, Dipartimento di Fisica, Piazza L. da Vinci 32, 20133 Milano, Italy \\ ${ }^{5}$ Center for Nano Science and Technology@PoliMi, Istituto Italiano di Tecnologia, I-20133 \\ Milan, Italy \\ cosimo.dandrea@polimi.it
}

\begin{abstract}
A time-resolved Diffuse Optical Tomography system based on multiple view acquisition, pulsed structured light illumination and detection with spatial compression is proposed. Reconstructions on heterogeneous tissue mimicking phantoms are presented.

OCIS codes: (170.6960) Tomography; (110.0113) Imaging through turbid media; (170.6920) Time-resolved imaging
\end{abstract}

\section{Introduction}

Diffuse Optical Tomography (DOT) is an imaging technique which aims at quantitatively reconstruct the spatial distribution of absorption and scattering properties of a biological tissue in vivo. Preclinical instrumentation is already available such as the case of optical mammography and oximetry systems. It is generally referred to Fluorescence Molecular Tomography (FMT) when fluorescent properties are measured and it is mainly used for small animal imaging.

A typical DOT/FMT system requires a dense distribution of source/detectors in order to increase the spatial information content and consequently improving spatial resolution and quantification capability. Moreover, a time-resolved detection on the ps scale, allows the possibility to retrieve absorption and scattering properties, to exploit temporal encoding of inclusions depth and to reconstruct fluorescence lifetime in the case of FMT. Finally, the use of different excitation/detection wavelength ranges allows one to discriminate tissue chromophores. This highly multidimensional problem leads to a huge data set which is not compatible with the acquisition/computational times generally required by a clinical and pre-clinical environment.

In the last years a new measurement scheme based on spatially-modulated illumination has been exploited in order to dramatically reduce the dimension of the data set while preserving spatial resolution [1-3]. Recently, this approach has been extended to the detection side using spatial light modulators (e.g. Digital Micromirror Device - DMD) [4]. In this case the output plane of the sample is imaged on the DMD hosting a spatial pattern, then all the light is focused on a single-element detector [5]. This principle is well known as "Single-Pixel camera". In the case of biological tissues, due to the scattering, the medium acts as a low-pass filter so, the use of a reduced set of spatial frequencies is justified for both the illumination and detection space, instead of a raster scanning scheme.

It is worth stressing that this approach shows relevant instrumental advantages. First of all the use of structure illumination allows to distribute high light power over a large area without exceeding light density safety limits and hence to reduce the acquisition time. Moreover, the use of a single pixel detection allows one to have higher performances (e.g. time resolution, spectral coverage) respect to parallel detectors (e.g. gated camera). Finally this approach allows to have global insights over the field of view (even if at lower spatial resolution) from first measurements.

In this work a fully tomographic approach for time-resolved DOT system based on a time-resolved single pixel camera and rotating view acquisition is proposed and experimentally validated on tissue mimicking phantoms with absorbing inclusions. The most relevant parameters related to tomographic capability and acquisition/computational times will be discussed.

\section{Materials and methods}

A scheme of the experimental set-up is shown in Fig.1. Light pulses at $650 \mathrm{~nm}$ (pulse width of tens of ps and rep. rate of $80 \mathrm{MHz}$ ) have been spectrally filtered by a tunable acousto-optic tunable filter from a supercontinuum mode-locked laser (SuperK Extreme, NKT). By means of a Digital Micromirror Device (DMD Discovery kit 1100 , Vialux) a spatially modulated light can be generated and subsequently imaged over a $3 \times 3$ $\mathrm{cm}^{2}$ area of the sample by an objective $(f=50 \mathrm{~cm})$. By means of a second lens $(f=60 \mathrm{~cm})$ the diffuse light exiting the sample, over a field of view of about $2 \times 2 \mathrm{~cm}^{2}$, is imaged on a second DMD (DMD Discovery 4100 , Vialux, Germany). The reflected light is focused on a $1 \mathrm{~mm}$ diameter optical fiber which on the other end is optically coupled to a photomultiplier (PMT) (HPM-100-50, Becker \& Hickl) which is connected to a Time-Correlated Single Photon Counting (TCSPC) board to obtain the temporal profile of the diffuse light. In order to obtain a 
Continuous Wave (CW) image of the DMD plane a low noise 16-bit cooled CCD camera (Versarray 512, Princeton Instruments) can be inserted by means of a flip mirror. This feature of the system is particularly useful to perform the pattern registration on the input and output side $[6,7]$. The whole measurement system is fully automatized and controlled by a home-made LabView software.

The tissue mimicking phantom is a cylinder $\left(\varnothing 20 \mathrm{~mm}\right.$, longer axis $50 \mathrm{~mm}$ ) made of an epoxy resin, $\mathrm{TiO}_{2}$ (as scatterer) and toner (as absorber) with an absorption of about $0.1 \mathrm{~cm}^{-1}$ and a reduced scattering coefficient of about $10 \mathrm{~cm}^{-1}$ both characterized with a validated time-resolved spectroscopy system [9]. Two cilindrical holes $(\varnothing 1.6 \mathrm{~mm})$ were drilled in the phantom as shown in fig. 1 . The two holes were then filled with a solution of water and calibrated Indian ink to obtain an absorption of about $0.5 \mathrm{~cm}^{-1}$. Then, a second measurement has been performed with different absorptions in the two holes $\left(0.5 \mathrm{~cm}^{-1}\right.$ and $\left.1 \mathrm{~cm}^{-1}\right)$.

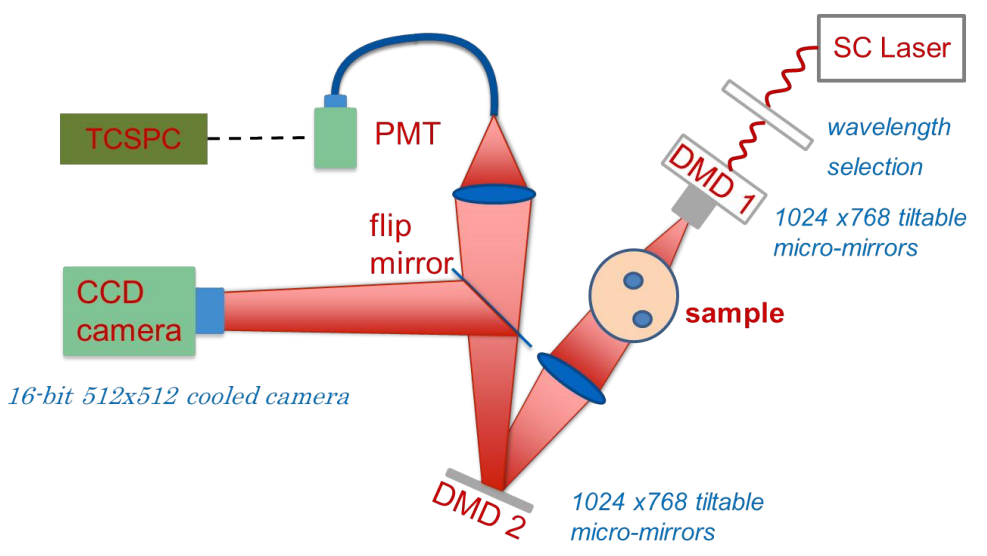

Fig. 1. Experimental set-up

The acquisition has been performed by a complete $360^{\circ}$ rotation of the sample with steps of $45^{\circ}$ (a total of 8 views). The sample is illuminated with a uniform square pattern and on the detection side $8 \times 8$ ordered WalshHadamard (WH) patterns have been used for compression. For every pattern the time-of-flight histogram has been acquired for $1 \mathrm{sec}$. For every WH pattern (ranging from -1 to +1 ) two positive patterns (ranging from 0 to +1 ) has been acquired. The instrumental response function (IRF) has been acquired by placing a paper sheet in place of the phantom.

The forward problem has been implemented using the finite-element based software TOAST++ [9]. Projectors functions based on OpenGL allow easily to project patterns directly on the cylindrical mesh to simulate both the illumination and the detection. The time-resolved Jacobian, taking into account the IRF, has been created with the adjoint method and then binned into a temporal window of about 500 ps on the rising edge of the temporal profile. The convolution between the direct and adjoint sources has been implemented using the Fast-Fourier Transform.

A Gauss-Newton method has been implemented for the minimization of the following merit function:

$$
\Psi\left(\boldsymbol{\mu}_{\boldsymbol{a}}\right)=\sum_{i=1}^{N_{\text {meas }}}\left(\frac{f_{n}\left(\boldsymbol{\mu}_{\boldsymbol{a}}\right)-y_{n}}{\sigma_{n}}\right)^{2}+\tau R_{T V}\left(\boldsymbol{\mu}_{\boldsymbol{a}}\right)
$$

Where $f_{n}\left(\boldsymbol{\mu}_{\boldsymbol{a}}\right)$ is the forward model computed at the nodes absorption $\boldsymbol{\mu}_{\boldsymbol{a}}, y_{n}$ is the gated measurement, $\sigma_{n}$ is the Poisson noise, $\tau$ is the hyper-parameter weighting the Total Variation (TV) regularizer $R_{T V}\left(\boldsymbol{\mu}_{\boldsymbol{a}}\right)$.

\section{Results and discussion}

Fig. 2 shows the tomographic reconstruction of the absorption coefficient at different vertical slices.

We observe a good quality reconstruction concerning both the 3D localization of the two inclusions and the spatial discrimination between the two inclusions. We note that the two inclusions are well localized and also the relative entities of the absorptions are well represented in the reconstruction. These preliminary results show that the quality of the data set by using the proposed approach based on the sampling in the illumination/detection space allows one to obtain a reconstruction quality at the state of the art.

It is worth mentioning that these preliminary reconstructions just employed a limited data set respect to the one which can be experimentally acquired. In particular, just one gate and one illumination pattern have been 
exploited. This is mainly due to the huge computational effort to handle the whole data set. In this perspective future work will be devoted to study the optimized data set in terms of illumination/detection patterns, number of views and gated images. This will bring to a reduction of the data set to allow shorter computational times. Presently the acquisition time of the data set exploited for the reconstruction reported in Fig. 2 required about $40 \mathrm{~min}$. This is quite long for many applications and in particular is much longer if the whole data set have to exploited. In this context a reduction of the data set and a reduction of the acquisition time of each image is desirable.

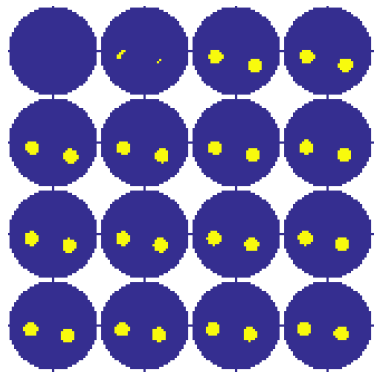

(a)

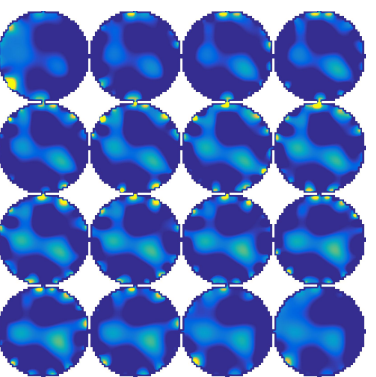

(b)

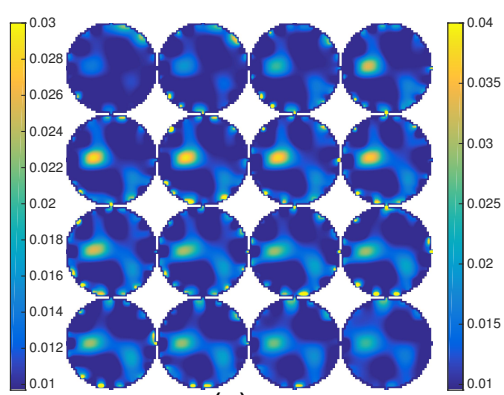

(c)

Fig. 2. (a) Position of heterogeneities. Absorption reconstruction with inclusions having the same absorption perturbation (b) and different perturbation (c). For each reconstruction 16 slices are displayed every $1 \mathrm{~mm}$ from $z=4 \mathrm{~mm}$ to $z=19 \mathrm{~mm}$. The top slice is not represented since it is not illuminated.

\section{Conclusions}

In conclusion, in this work a time-resolved DOT system based on rotating view acquisition and data sampling in the compressed illumination/detection space has been proposed and implemented. By using appropriate wavelength filters the proposed system can be easily adapted to perform FMT. We believe the proposed approach based on the sampling in the spatial frequency domain represents an efficient wide field DOT instrumentation to reduce the acquisition time while preserving as much as possible the spatial information content. Future works will be devoted to optimize the data set in order to reduce the acquisition times and in general the dimensionality of the data set.

This work was partially supported by Cariplo Foundation under Grant N. 2013-0615 and by the Royal Society International Exchanges 2014/R1.

\section{References}

[1] Bassi, A., D’Andrea, C., Valentini, G., Cubeddu, R., Arridge, S.., , "Temporal propagation of spatial information in turbid media," Opt. Lett. 33(23), 2836-2838 (2008)

[2] Cuccia, D. J., Bevilacqua, F., Durkin, A. J.., Tromberg, B. J., "Modulated imaging: quantitative analysis and tomography of turbid media in the spatial-frequency domain.," Opt. Lett. 30(11), 1354-1356 (2005)

[3] Venugopal, V., Chen, J., Lesage, F., Intes, X., "Full-field time-resolved fluorescence tomography of small animals," Opt. Lett. 35(19), 3189-3191 (2010)

[4] Pian, Q., Yao, R., Zhao, L., Intes, X., "Hyperspectral time-resolved wide-field fluorescence molecular tomography based on structured light and single-pixel detection.," Opt. Lett. 40(3), 431-434, OSA (2015)

[5] Duarte, M. F., Davenport, M. A., Takhar, D., Laska, J. N., Sun, T. S. T., Kelly, K. F.., Baraniuk, R. G., "Single-Pixel Imaging via Compressive Sampling," IEEE Signal Process. Mag. 25 (2008).

[6] ] Ducros, N., Bassi, A., Valentini, G., Schweiger, M., Arridge, S.., D’Andrea, C., "Fluorescence molecular tomography of an animal model using structured light rotating view acquisition .," JBO 18(2), 02050 (2013)

[7 ] Ducros, N., Bassi, A., Valentini, G., Canti, G., Arridge, S.., D’Andrea, C., "Multiple-view fluorescence optical tomography reconstruction using compression of experimental data.," Opt. Lett. 36(8), 1377-1379 (2011)

[8] Konugolu Venkata Sekar, S., Dalla Mora, A., Bargigia, I., Martinenghi, E., Lindner, C., Farzam, P., Pagliazzi, M., Durduran, T., Taroni, P., Pifferi, A., and Farina, A., "Broadband (600-1350 nm) Time Resolved Diffuse Optical Spectrometer for Clinical Üse," IEEE'J. Sel. Top. Quantum Electron. 22(3), 406-414 (2015).

[9] M. Schweiger and S. Arridge, "The Toast ++ software suite for forward and inverse modeling in optical tomography," J. Biomed. Opt. 19, 1-15 (2014). 